\title{
Radargrammetry of Opposite-Side Stereo Magellan Synthetic Aperture Radar on Venus
}

\author{
Hoonyol Lee, Joanna V. Morgan, and Michael R. Warner \\ Department of Earth Science and Engineering \\ Imperial College London \\ London SW7 2AZ, United Kingdom \\ hoonyol.lee@imperial.ac.uk
}

\begin{abstract}
Radargrammetry of opposite-side stereo SAR is demonstrated using Magellan SAR images of Venus. Large areas of gentle slope can be successfully matched to generate DEM using conventional stereo-match algorithm while high to moderate relief regions need further treatment using shape-fromshading. The initial stereo-match points are classified into three categories; GOOD, $B A D$, and $T O P O$, and different methods are applied to different regions to iteratively improve DEM.
\end{abstract}

Keywords - opposite-side,stereo, synthetic aperture radar, Magellan

\section{INTRODUCTION}

Opposite-side stereo synthetic aperture radar (SAR), using left- and right-looking SAR intensity images, gives higher radargrammetric sensitivity to height than same-side stereo SAR, but is difficult to match two SAR images to generate digital elevation model (DEM) due to severe topographic image distortion. Conventional stereo matching algorithm fails on high to moderate relief region. Some previous studies recognized its availability on areas of gentle slope on the earth's surface [1]. However, the area suitable for this purpose is very limited because of vegetation and rapidly erosion on the earth's surface.

Magellan SAR images obtained in early 1990s [2] have shown that large part of Venus' surface is covered by fine sediment and the surface erosion rate is very low. There are far more areas of radar-dark gentle slope region on Venus than the earth's surface. The radargrammetric capability of oppositeside SAR images obtained over $50 \%$ of the Venusian surface can be substantial when compared to the same-side stereo SAR images available on $21 \%$ of the surface.

In this paper, we present the radargrammetry of oppositeside Magellan SAR images on Venus. Two images are matched using initial conventional stereo matching process. The matching points are classified into three categories: $G O O D, B A D$ or $T O P O$, based on the analysis of three parameters induced from stereo matching: signal-to-noise ratio of normalized cross-correlation(SNR), X-, Y-disparity(image shift between two images in horizontal and vertical direction). The initial stereo match result is then refined iteratively by applying different treatments to different categories.

Previous effort has been reported to process the oppositeside SAR images for stereo-viewing by inverting brightness and correcting geometry [3]. However, the radarclinometry or shape-from-shading technique is often very unstable because of the mathematically ill-posed nature of the problem, especially for non-homogeneous region. The classification of the initial stereo match result used in this paper will reduce possible error and computation time by selectively applying local shapefrom-shading to TOPO region only.

\section{INITIAL STEREO-MATCH AND CLASSIFICATION}

A conventional computer-based stereo-matching process is applied to opposite-side stereo SAR images. A small patch from the master image is taken to be searched around the slave image, producing normalized cross-correlation (NCC) values ranging $[-1,1]$. Three parameters can be produced from image matching algorithm: X-, Y-disparity and SNR. The $\mathrm{x}, \mathrm{y}-$ disparity between two image pair is determined by picking the shift point that gives the highest NCC result. The SNR is defined to represent of the "peakness" of the NCC distribution over image shift. These three parameters can is then used for assessment of match quality and classification.

\section{A. $X$ - and $Y$-disparity}

In an ideal case where stereo images are in the same SAR coordinates ( $\mathrm{X}$ in range direction, $\mathrm{Y}$ in azimuth direction and two orbits are parallel with each other), height should be embedded only in range direction and the azimuth direction should bear no topographic information. Only X-disparity is a function of height in this case while $\mathrm{Y}$-disparity remains constant. Y-disparity found in stereo match imposes very strong restriction for match qualification.

Magellan mosaic SAR images are best obtainable in FMAP format. Errors from image mosaic can be several hundred meters in azimuth direction even if the refined altimeter and orbit information is used. This error will appear as a constant Y-disparity within an orbit and can be compensated easily. More complicating fact is that Magellan SAR images are not in SAR coordinates or parallel with each other. Magellan stereo SAR images show different orbit inclination angles. X$\operatorname{disparity}(d x)$ will be affected by the orbit inclination angles ( $\delta_{1}$ and $\delta_{2}$ ) measured as a footprint of the orbit on the image plane and defined positive anti-clockwise from vertical $\mathrm{Y}$-axis. $\operatorname{Height}(h)$ also smear into Y-disparity $(d y)$ as:

$$
\begin{aligned}
& d x=h\left(\cot \theta_{1}^{0} \cos \delta_{1}-\cot \theta_{2}^{0} \cos \delta_{2}\right)+d x_{0} \\
& d y=-h\left(\cot \theta_{1}^{0} \sin \delta_{1}-\cot \theta_{2}^{0} \sin \delta_{2}\right)+d y_{0},
\end{aligned}
$$

This research is funded by ROPA, PPARC, UK 
where $\theta_{1}^{0}$ and $\theta_{2}^{0}$ are the nominal incidence angles of two images measured positive anti-clockwise. They are the same signs in case of same-side stereo pair while different in case of opposite-side stereo pair. $d x_{0}$ and $d y_{0}$ represent constant image shifts originated from image mosaic error during FMAP generation as discussed above. If the orbit inclination angles are zero, X-disparity will be of the same form which can found in many literatures[1-3] and Y-disparity becomes zero.

\section{B. SNR}

One way of representing quality of peak selection out of the NCC map generated from stereo-match is the signal-to-noise ratio (SNR) defined as

$$
S N R=\frac{(1+\text { peak } N C C)}{(1+\text { average } N C C)},
$$

which will have value range of $[1, \infty)$. SNR shows very high correlation to image contrast, which can be defined as a standard deviation of the intensity distribution within the selected image patch. A high contrast image patch shows high SNR. As an extreme case when the intensity distribution is constant then the image contrast will be zero and the value of cross-correlation will be constant, producing the lowest SNR value possible, i.e. 1. NCC is not even defined in this case and should be dealt with to prevent computational overflow.

\section{Classification using $S N R, X$-, $Y$-disparity into GOOD, $B A D$, and $T O P O$}

Three parameters induced from the initial matching procedure (X-, Y-disparity and SNR) can be used to qualify and classify the stereo matching points. X-disparity is used to limit the height range by intentionally allowing a large search area over the slave image during the initial stereo match algorithm so that any matching point well beyond the expected height range should be claimed as bad matching point. As Xdisparity is largely a function of height when orbit inclination is small, as seen in (1), it usually serves as a very weak restriction on assessment of image matching quality. On the contrary, Y-disparity can serve as a strong restriction when orbit inclination and mosaic error is considered properly. The acceptable Y-disparity range can vary with orbit by orbit when dealing with large area mosaicked with several orbits. It also is a weak function of height as shown in (1). The search area in $Y$ direction should be set larger than the expected Y-disparity so that it serves as a criterion for image matching quality. SNR is another important parameter. Matching points with low SNR are typically in low contrast area of the intensity image and should be discarded due to possible error in NCC peak detection.

We use these three parameters to classify the initial stereo image matching points. Three distinct types of surface can be identified. Gently sloping area with slope less than a few degrees and with good image contrast will satisfy all three restrictions. It shows X-, Y-disparity, and SNR within reasonable range. The quality of DEM is good in these areas and thus categorized into GOOD. There are areas of very low contrast where topography is very gentle. These areas show typically low SNR, and named as $B A D$. Areas of high to moderate slope have high image contrast due to layover, radar-bright foreshortening, radar-dark enlargement, and shadow so that they usually show high SNR. However, they tend to have extreme value of $\mathrm{X}$-disparity and often have very random Y-disparity as well due to topographic distortion. This area is categorized as TOPO. An example of classification used in this study is depicted as a Venn diagram in Fig. 1.

\section{ITERATIVE DEM IMPROVEMENT}

Different treatments will be applied to different categories classified from the initial stereo-match result to improve the DEM quality iteratively.

\section{A. GOOD: Gentle slope with good X-, Y-disparity and SNR}

The quality of image-matching points classified into $G O O D$ is mostly satisfactory for DEM generation. A further improvement in spatial resolution can be achieved reducing the size of the image patch. A smaller search region over the slave image can be used to cut the computational cost, but sufficiently large search area is still recommended for further classification.

\section{B. BAD: Gentle slope with poor SNR}

$B A D$ points occur at low image contrast area and have very low SNR. Image contrast can be increased by using larger image patch to attract more surface features. This will increase the image contrast and SNR at a cost of spatial resolution.

\section{TOPO: High to moderate slope with poor $X$ - or $Y$ - dispartiy but sill good SNR}

Poor X- and/or Y-disparity in TOPO points result from topographic distortion of image, i.e. the difference of image features between two stereo images. For the case of oppositeside stereo SAR, this feature mismatch becomes extremely large because a foreshortening radar-bright slope in one image, for example, will be an enlarged radar-dark slope in the other image. This topographic distortion should be dealt with for a successful implementation of radargrammetry on high to moderate relief using opposite-side SAR.

One possible solution to this is shape-from-shading, or radarclinometry. As we have at least two image, we are developing a new method to use localized shape-from-shading in context of radargrammetry. From the master image, we estimate backscattering function from which we can convert intensity of the master image pixel into local slope. The corresponding pixel length and intensity of the slave image is then calculated according to the estimated backscattering function of the slave image. Therefore, the master image patch is simulated to the slave image geometry, and is used for 
searching over the actual slave image to find best match. The global error propagation, which is a typical error source of radarclinometry, will be localized within image patch.

Radar backscattering function of incidence angle is usually unknown and should be estimated from the SAR image itself. Non-homogeneous features are often included in the SAR image of interest, which complicate the application of shapefrom-shading. Other non-SAR experiment such as radiometer or altimeter measurement from Magellan spacecraft provided surface reflectivity and emissivity. However, no data is available with spatial resolution comparable to SAR images.

Let's assume that the image patch selected for stereo matching is homogeneous and the backscattering function is symmetric. We also assume Gaussian slope distribution with average slope $\bar{\alpha}$ and standard deviation $\sigma_{\alpha}$. When this surface is viewed from SAR geometry, the distribution function of slope featured in SAR ground range will be:

$$
G(\alpha)=\frac{1}{\sqrt{2 \pi} \sigma_{\alpha}} \exp \left(-(\alpha-\bar{\alpha})^{2} / 2 \sigma_{\alpha}^{2}\right)\left(1-\tan \alpha / \tan \theta^{0}\right) .
$$

This function shows slightly skewed Gaussian shape with mode(most frequent slope) shifted inversely proportional and opposite to the nominal incidence angle, and proportional to the standard deviation of slope.

Intensity values( $\mathrm{DN})$ in the Magellan SAR images were adjusted using an empirically derived Muhleman's backscattering function[2] and then converted into logarithmic scale. This makes the relationship between DN and slope very much linear over large part of the visible slope range. If we can assume a linear relationship between $\mathrm{DN}$ value and slope such as $D N=a \alpha+b$, then the intensity distribution function can be derived using simple linear algebra. This distribution function will have very similar form to the slope distribution in (3). This means that we can derive the two linear backscattering coefficients $a$ and $b$ from the local distributions of the two SAR intensity images.

The shape of SAR intensity distribution also gives a good measure whether the area is homogeneous or not. If the intensity distribution is unimodal, i.e., if it has only one distinct peak, and the skewness is small, then we can assume the image patch is homogeneous. If the intensity distribution is bimodal or multimodal, then the image patch is a mixture of at least two different surface types. Therefore, the modality of intensity distribution indicates the degree of surface non-homogeneity so that it can be used for a shape-from-shading procedure.

The backscattering function estimated in this way serves as a good initial starting value for the iterative search methods such as genetic algorithm where backscattering coefficients evolves with generations to result best match criteria. The use of genetic algorithm is being tested and compared with other search methods in its efficiency and convergence.

\section{EXAMPLE AND CONCLUSION}

An example of opposite-side stereo SAR in Lullin impact crater on Venus is shown in Fig. 2. Large area of the radar-

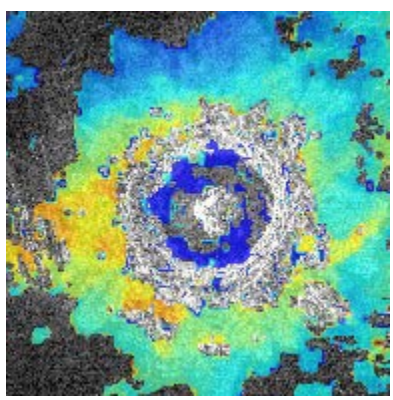

(a) $G O O D$

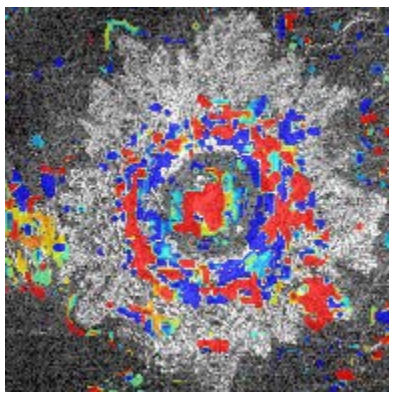

(c) $\mathrm{TOPO}$

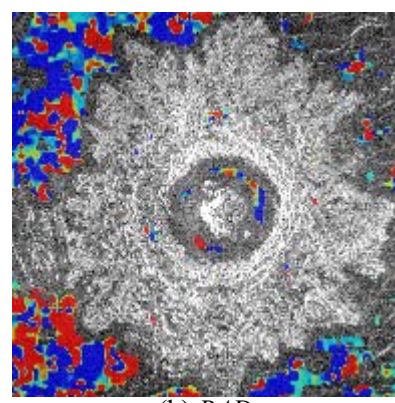

(b) $B A D$

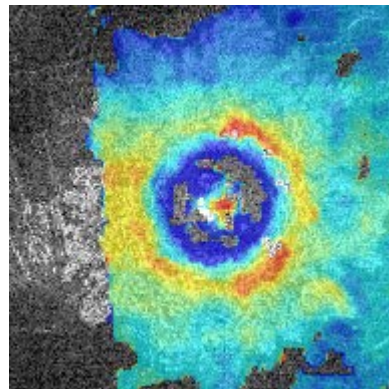

(d) Same-side GOOD
Figure 2. Pseudocolor DEM of Lullin impact crater on Venus from opposite-side stereo $\operatorname{SAR}(60 \mathrm{~km} \times 60 \mathrm{~km})$. The initial stereo match result is classified into (a)GOOD, (b)BAD and (c)TOPO. (d) shows same-side stereo SAR result with missing orbit on the left part. Height difference between blue to red is about $1 \mathrm{~km}$. Background image is the left-look SAR.

bright crater ejecta, having gentle slope and high image contrast, shows good result from the conventional stereomatching algorithm, and is classified as GOOD accordingly(a). The surrounding radar-dark plain and the crater floor show very low image contrast resulting in low SNR, and classified as $B A D$ (b) to be reprocessed with larger image patch. Central peak and crater wall with high slope is classified as TOPO for further treatment with localized shape-from-shading (c). DEM from the same-side stereo SAR pair is shown in (d) for comparison.

This preliminary result shows that opposite-side stereo SAR can be used to generate good quality DEM of gentle topography which is abundant on the planet Venus. Further research is still ongoing to improve DEM quality of high to moderate relief. The use of opposite-side stereo SAR could be beneficiary especially to planetary applications such as the upcoming Cassini mission on Titan. This technique will also provide operational flexibility for the future planetary- and earth-bound SAR missions.

\section{REFERENCES}

[1] T. Toutin, "Opposite-side ERS-1 SAR Stereo mapping over rolling topography", IEEE Trans. Geosci. Rem. Sens., vol. 34, no. 2, March 1996, pp. 543-549

[2] J. P. Ford, J. J. Plaut, C. M. Weitz, T. G. Farr, D. A. Senske, E. R. Stofan, G. Michaels, and T. J. Parker, Guide to Magellan Image Interpretation, NASA, JPL, November 1993.

[3] J. K. Fullerton, F. Leberl, and R. E. Marque, "Opposite-side SAR image processing for stereo viewing", Photo. Eng. Rem. Sens., vol. 52, no. 9 , September 1986, pp. 1487-1498. 